\title{
A Novel Right Ventricle Segmentation Approach from Local Spatio-temporal MRI Information
}

\author{
Angélica Maria Atehortúa Labrador, Fabio Martínez, \\ and Eduardo Romero Castro \\ CIM@LAB, Universidad Nacional de Colombia, \\ Ciudad Universitaria Cra 30 N 45-03 Facultad de Medicina Centro de telemedicina, \\ Bogotá, Colombia \\ \{amatehortual, fmartinezc, edromero\}@unal.edu.co \\ http://cimlaboratory.com/
}

\begin{abstract}
This paper presents a novel method that follows the right ventricle (RV) shape during a whole cardiac cycle in magnetic resonance sequences (MRC). The proposed approach obtains an initial coarse segmentation by a bidirectional per pixel motion descriptor. Then a refined segmentation is obtained by fusing the previous segmentation with geometrical observations at each frame. A main advantage of the proposed approach is a robust MRI heart characterization without any prior information. The proposed approach achieves a Dice Score of 0.62 evaluated over 32 patients.
\end{abstract}

Keywords: Right Ventricle Segmentation, Cardiac MRI Cine, Local Motion Models, Structural Information.

\section{Introduction}

Cardiovascular diseases (CVDs) are world wide one of the principal causes of death and disability [1. An accurate quantification of the right ventricular structure and function has become important to support the diagnosis, prognosis and evaluation of several cardiac diseases and also to complement the typical analysis of the left ventricular function [23. Currently, most common methods, for assessing the heart, are based on quantification and characterization of patterns during a Cardiac Magnetic Resonance Imaging (CMRI) [4]. Such methods are widely used to analyze, diagnose and even prognose certain heart diseases. Among the evaluated heart variables, the most common are the ventricular chamber sizes, volumes and masses at each cardiac phase, ventricular function and correlation flow [5]. A proper RV analysis demands an accurate 3D temporal segmentation, specifically endocardial and epicardial contours. Typically such task is carried out by expert cardiologists who perform manual delineations that may take $18.9 \pm 4 \mathrm{~min}$ [6] per case, introducing high inter-and-intra observer variability 37. Therefore, automatic segmentation methods are appealing to obtain more accurate RV temporal-segmentation. Nevertheless, several challenges arise because of the complex RV geometry shape and high non-linear heart motion

J. Ruiz-Shulcloper and G. Sanniti di Baja (Eds.): CIARP 2013, Part II, LNCS 8259, pp. 206-213, 2013.

(C) Springer-Verlag Berlin Heidelberg 2013 
during diastole and systole transition. In addition, RV fuzzy edges and random acquisition noise make more challenging the RV segmentation [6].

Several state-of-the-art methods have been proposed for automatic RV segmentation, most of them based on the use of strong structural and appearance priors that adjust the shape w.r.t a set of samples. In this sense, these methods use mainly statistical shape models, multi-atlas strategies and deformable approximations [6]. These strategies are strongly dependent on how data is learned to build up the prior. However, accurate quantification of certain variables like the ejection fraction depends on the shape changes, particularly important in pathological cases. In addition, such approaches pay a high price when mapping the prior to the MR since the metrics is usually noisy because of the dependency of intensity variations or the pixel spatial distribution to represent the heart, facts that may lead to inconsistent segmentations 6 .

On the other hand, methods with no prior are based on appearance and temporal MRI information. Cocosco et al. [8] describe the segmentation of both the left ventricle $(\mathrm{LV})$ and right ventricle $(\mathrm{RV})$, by a simple temporal RoI estimation of major motions and then a voxel classification is performed between RV and LV using morphological operations. However, the simplicity of the temporal descriptor, a simple subtraction between consecutive frames, turns out to be very noisy. In addition, Wang et al. 9] capture information that is shared during the sequence and merge it with a spatial within-frame descriptor, based on a classical isodata algorithm. Nonetheless, RV segmentation may easily overflow the actual borders.

The main contribution of this work is a fully automatic method that uses no prior at all and delineates the RV endocardium contour in 4D MR sequences. The strategy uses both a heart motion descriptor and an estimation of RV shape for each frame of the sequence. Firstly a robust per pixel motion model is introduced to highlight the edges with major changes along the sequence, under the hypothesis that heart is the organ with larger motion. Afterwards, a conventional isodata strategy estimates the heart shape which is superimposed to the edges computed from the motion estimation. The final delineation is set to the intersection between those edges and estimated heart shape. The following section describes the proposed segmentation approach. In section 3, the evaluation and results. Finally in section 4 is presented the discussion on the results obtained and some conclusions.

\section{Methodology}

The strategy herein proposed is capable of capturing the temporal RV contours from a spatio-temporal MRI characterization. As widely acknowledged, heart motion is the main biomarker in cardiology, allowing by itself an appropriate assessment of cardiac function [10. Hence, the approach starts by coding temporal MRI information with a bidirectional per-pixel motion descriptor [11]. A coarse heart segmentation is initially obtained from that estimated cardiac motion. This segmentation is corrected using geometrical observations from the 


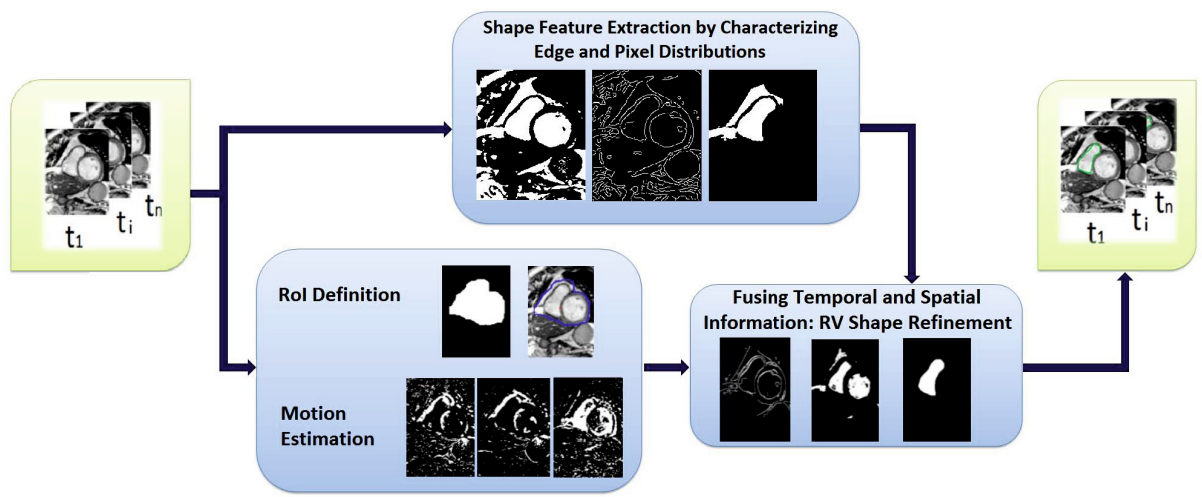

Fig. 1. The proposed method. A motion descriptor is computed for the whole MRI cardiac cycle, which is then adjusted to the edged and spatial estimation found at each frame in the estimated shape.

estimated shape. The pipeline of the proposed approach is illustrated in Figure 1 and described in the following subsections.

\subsection{Motion Estimation}

The heart is the organ whose vital function amounts to a constant motion. The proposed strategy starts by estimating the cardiac movement with a bidirectional local motion descriptor. For doing so, a temporal median sets the elements with less motion during the sequence by recursively updating the frame median, as follows $M_{t}(x)=M_{t-1}(x)+\operatorname{sgn}\left(I_{t}(x)-M_{t-1}(x)\right)$, where $M_{t}(x)$ represent the median and $I_{t}(x)$ the frame at time $t$ for each pixel $x$. Using such recursive median, a likelihood measure $\Delta_{t}$ sets those pixels in movement at each instant $t$ as $\Delta_{t}=\left|M_{t}(x)-I_{t}(x)\right|$. This last term is in due turn regularized by a cumulated variance of the motion history, as: $V_{t}(x)=V_{t-1}(x)+\operatorname{sgn}\left(N \times \Delta_{t}(x)-V_{t-1}(x)\right)$. This descriptor is highly noise robust and computes the per-pixel temporal variation that allows to classify the RV. Specifically, At the End of the Diastole, when the heart is maximally expanded, pixel candidates should meet two conditions: the pixel motion is larger than an accumulated temporal variance under the restriction that the movement must span an important percentage of the cardiac cycle. Such relationship is represented by a simple thresholding as

$\widehat{B S}_{t}^{(D)}(x)=\Delta_{t}(x) \geq V_{t}(x)$. In contrast, at the Systole, the heart contraction is maximum and the motion is practically null so that this phase constructs a very steady history of the cardiac flow. After the semilunar valves open, blood flows out the ventricle with an important change that is very likely detected by the motion estimation algorithm. The heart edges are thus calculated from pixels with major motion by comparing the likelihood measure with a learned scalar parameter $\tau$ as: $\widehat{B S}_{t}^{(S)}(x)=\Delta_{t}(x) \geq \tau$. 
Classically, local motion descriptors [11] are usually unidirectional recursive algorithms, but in this case the first iteration yielded a very blurry estimation of the heart contour at the End-of-Diastole. As the recursive accuracy depends on the captured motion history, the descriptor is herein bidirectionally run, i.e., forward and backward as $B S_{t}(x)^{(D, S)}=\alpha{\widehat{B S_{t}}}^{(D, S)}(x)+(1-\alpha){\widehat{B S_{N-t}}}^{(D, S)}(x)$, where $\alpha$ is an important parameter defined as $\frac{t}{N}$ and $N$ is the number of frames. Once motion has been thresholded, morphological operators groups up pixels associated with movement [1].

\subsection{Shape Feature Extraction by Characterizing Edge and Pixel Distributions}

The previous motion estimation produces a coarse shape segmentation and serves also to define a Region of Interest (RoI). The aim of this second phase is to construct another complementary shape approximation, using exclusively spatial observations. A first approximation to such heart shape consisted in finding a RoI that consistently surrounded the heart, as the spatial region with larger temporal motion at each time step. Within such RoI, heart ventricles are estimated from two complementary measurements: a global shape clustering and an edge extraction.

Firstly a global shape description of the ventricles was herein obtained by a classical isodata algorithm [12 that is used to separate the intensities corresponding to the myocardium and the cardiac chambers. The cardiac wall or myocardial tissue is segmented and therefore the right and left heart chambers serve as a reference frame of the right and left ventricles.

On the other hand, ventricle edges are estimated from the MRI RoI by using a conventional Canny filter [13. In the apical slices however, while the LV is still differentiable, RV edges are very blurred (as shows Figure 2). Overall, edges in apical slices are considered as part of the LV. Estimations of RV edges are performed from the previous motion estimation provided that such edge is not already part of the previously defined LV edges.

\subsection{Fusing Temporal and Spatial Information: RV Shape Refinement}

During certain phases of the cardiac cycle, some boundaries of the heart were not properly segmented. Two fusion strategies were herein implemented to cope with such issue: 1) a first approach fused the spatial information obtained from the temporal information with the edge estimation and the isodata algorithm 2) a second strategy fused the temporal and isodata informations, but using exclusively the left ventricle isodata information. This second approach was particularly useful to segment the right ventricle at the apex level. For the first fusion strategy, the two edge representations are fused by simply summing and normalizing. The final shape is in this case outlined by intersecting both the RV 


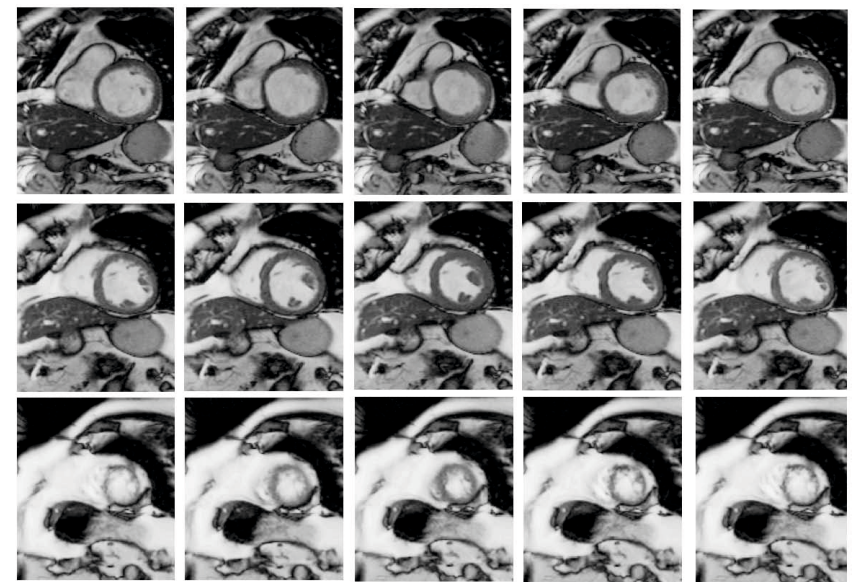

Fig. 2. The variability of the RV shape, from basal (top row) to apical (bottom row), and from left to right for the whole cardiac cycle, being the first column the End-ofDiastole and the mid column the End-of-Systole

shape estimated from the isodata information and edges. For the second strategy, it was applied a simple difference between the temporal heart segmentation and the spatial LV segmentation obtained by the isodata algorithm so that the remaining pixels then correspond to the RV. Finally, isolated pixels are always removed by simple opening and closing operators.

\subsection{Data}

The evaluation of the proposed approach was performed over a public Cardiac MRI dataset 314 with 32 patients split into two subsets: training and test data set, which are specified by the authors of the dataset. For evaluation, the obtained segmentation was submitted to the RVSC [15] which sends back the results. Training data consisted in a set of 16 cardiac MRI, half split into men and women, with an average age of $51 \pm 12$ years. For test data was split into 3 women and 13 men cases, respectively, with and average age of $48 \pm 18$ years. The recorded patients were diagnosed with several cardiac pathologies like myocarditis, ischaemic cardiomyopathy, arrhythmogenic right ventricular dysplasia (ARVD), dilated cardiomyopathy, hypertrophic cardiomyopathy, Aortic stenosis, cardiac tumour, left ventricular and ejection fraction assessment. Each MR sequence was captured in the short-axis with 1.5 Tesla, in a plane resolution of $1.3 \mathrm{~mm}$ and a between-slice distance of $8.4 \mathrm{~mm}$. The epicardium and endocardium of $32 \mathrm{MR}$ sequences were manually delineated by an expert cardiologist. Trabeculae and papillary muscles were included in the RV cavity.

\section{$3 \quad$ Evaluation and Results}

Figure 3 illustrates the good performance of the method in cardiac MRI sequences. The green contour corresponds to the result obtained by the presented 

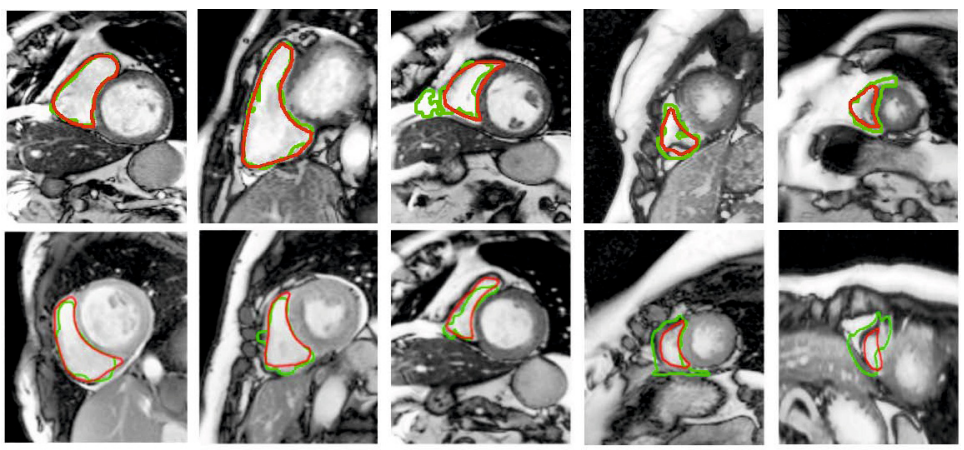

Fig. 3. Example of RV segmentations with several cases, including the End-of-Diastole (firts row) and End-of-Systole (second row). The ground truth is the red line and the green line is the automatic segmentation. As expected, better results were observed at the basal slices (first column).

method, while the ground truth is drawn in red. As expected, failures are mainly present in apical images because of the fuzzy borders and small RV area.

Quantitative technical evaluation was performed using the most classical metrics described in the literature: Dice Score (DSC) measure 16] and Hausdorff distance (HD) [17]. An overlap DSC measure is defined as: $D S C(A, B)=\frac{2(A \cap B)}{A+B}$, where $A$ and $B$ represent the obtained area and the expert ground truth, respectively. On the other hand, the Hausdorff measure $H(A, B)$ computes the maximum distance between two sets of points as $\max (H(A, B), H(B, A))$ and

$H(A, B)=\max _{a \in A} \min _{b \in B}\|a-b\|_{2}^{2}$. In this case, each set of points represents the organ surface. This measure allows to indirectly assess the compactness of the segmentation. A clinical performance was also assessed as the ejection fraction $(\mathrm{EF})$.

Table 1. Performance of the proposed approach for training data using Dice Score (DSC) and Hausdorff distance (HD) over the Endocardium contour

\begin{tabular}{|c|c|c|}
\hline & $\begin{array}{c}\text { DSC } \\
\text { mean (std) }\end{array}$ & $\begin{array}{c}\text { HD (in mm) } \\
\text { mean (std) }\end{array}$ \\
\hline \hline End-of-Diastole (ED) & $0.66(0.22)$ & $20.66(13.00)$ \\
\hline End-of-Systole (ES) & $0.54(0.26)$ & $27.72(23.45)$ \\
\hline
\end{tabular}

Quantitative results were only evaluated at End-of-Diastole (ED) and Endof-Systole (ES) since these two states are the most important to clinical quantification [18. As baseline it was taken the work proposed by Wan et. al [9], which until now represents the best strategy to segment the RV without prior. Table 1 summarizes the obtained performance for training data sequences in ED 
and ES times. The proposed approach clearly outperforms the baseline method in terms of overlapping and compactness in both cardiac states. As expected a much better segmentation is obtained at the ED because the MRI frame quality allows a better quantification. Although, at the ES many times the poor MRI contrast leads to a quite fuzzy RV edges, the proposed approach outperforms the state-of-the-art approach. Table 2 summarizes the performance obtained by our approach over the test data. Although the obtained score errors are slightly larger for the RV segmentations, the proposed approach properly captures the shape variability and is easily adapted to new RV shapes since it only depends on the particular MRI observations.

Table 2. Performance of the our RV segmentation method for the Test data set using Dice Score (DM) and Hausdorff distance (HD) over the Endocardium contour

\begin{tabular}{|c|c|c|c|c|}
\hline & \multicolumn{2}{|c|}{ Our approach } & \multicolumn{2}{c|}{ Baseline } \\
& $\begin{array}{c}\text { DSC } \\
\text { mean (std) }\end{array}$ & HD (in mm) & DSC & HD (in mm) \\
mean (std) & mean (std) & mean (std) \\
\hline \hline ED & $0.72(0.29)$ & $16.17(16.48)$ & $0.63(0.32)$ & $22.89(25.01)$ \\
\hline ES & $0.51(0.31)$ & $27.47(27.96)$ & $0.50(0.34)$ & $27.99(24.97)$ \\
\hline
\end{tabular}

Finally, it was calculated the mean error for the ejection fraction, defined as error $=\sum_{p=1}^{N} E F p_{\text {auto }}-E F p_{\text {manual }}$, where an error of 0.36 was obtained over the whole data set (32 patients). Although the error index shows an acceptable performance, some important noise sources, such as the inter-and-intra high variability of RV shape, the fuzzy edges and the complex heart motion, are not properly captured by our method. Nevertheless, the approach herein presented shows appropiate RV segmentations using an strategy based principally in temporal characterization. This approach outperform state-of-the-art methods that use only appearance and temporal observations for each sequence [89].

\section{Conclusions}

In this paper it was introduced a new strategy to segment the right ventricle in MR sequences. The proposed mixed approach uses spatio-temporal observations and produces reliable RV segmentations. A great advantage of the proposed approach is its independency of any prior heart shape, facilitating the capture of dynamic and shape heart variability, which could be associated to specific cardiac pathology. In future work, the method could extend to 3D processing and further validation with a larger data set will be performed.

\section{References}

1. Roger, V., Go, A., Lloyd-Jones, D., Adams, R., Berry I, J.: Heart disease and stroke statisticts - 2012 update: A report from the american heart association. Circulation 125, 200-220 (2012) 
2. Haddad, F., Doyle, R., Murphy, D., Hunt, S.: Right ventricular function in cardiovascular disease, part ii: pathophysiology, clinical importance, and management of right ventricular failure. Circulation 117, 1717-1731 (2008)

3. Caudron, J., Fares, J., Lefebvre, V., Vivier, V., Petitjean, C., Dacher, J.: Cardiac mri assessment of right ventricular function in acquired heart disease: factors of variability. Acad. Radiol. 19, 991-1002 (2012)

4. Baur, L.: Magnetic resonance imaging: the preferred imaging method for evaluation of the right ventricle. The International Journal of Cardiovascular Imaging 24, 699-700 (2008)

5. Haddad, F., Hunt, S., Rosenthal, D., Murphy, D.: Right ventricular function in cardiovascular disease, part i: Anatomy, physiology, aging, and functional assessment of the right ventricle. Circulation 117, 1436-1448 (2008)

6. Petitjean, C., Dacher, J.: A review of segmentation methods in short axis cardiac mr images. Med. Image Anal. 15, 169-184 (2011)

7. Caudron, J., Fares, J., Vivier, P., Lefebvre, V., Petitjean, C., Dacher, J.: Diagnostic accuracy and variability of three semi-quantitative methods for assessing right ventricular systolic function from cardiac mri in patients with acquired heart disease. Eur. Radiol., 2111-2120 (2011)

8. Cocosco, C., Niessen, W., Netsch, T., Vonken, E., Lund, G., Stork, A., Viergever, M.: Automatic image-driven segmentation of the ventricles in cardiac cine MRI. J. Magn. Reson. Imaging 28, 366-374 (2008)

9. Wang, C., Peng, C., Chen, H.: A simple and full automatic right ventricle segmentation method for 4-dimensional cardiac MR images. In: RV Segmentation Challenge in Cardiac MRI MICCAI 2012 (2012)

10. Punithakumar, K., Ayed, I.B., Islam, A., Goela, A., Ross, I.G., Chong, J., Li, S.: Regional heart motion abnormality detection: An information theoretic approach. Medical Image Analysis 17(3), 311-324 (2013)

11. Manzanera, A., Richefeu, J.C.: A new motion detection algorithm based on $\sigma-\delta$ background estimation. Pattern Recogn. Lett. 28(3), 320-328 (2007)

12. Ridler, T., Calvard, S.: Picture thresholding using an iterative selection method. IEEE Transactions on Systems, Man and Cybernetics 8(8), 630-632 (1978)

13. Canny, J.: A computational approach to edge detection. IEEE Transactions on Pattern Analysis and Machine Intelligence PAMI-8(6), 679-698 (1986)

14. Download MRI Data from RV segmentation Challenge MICCAI 2012 (2012), http://www.litislab.eu/rvsc/rv-segmentation-challenge-in-cardiac-mri/ download

15. Evaluation Framework Right Ventricle Segmentation (2012), http://www.litislab.eu/rvsc

16. Dice, L.R.: Measures of the amount of ecologic association between species. Ecology 26(3), 297-302 (1945)

17. Huttenlocher, D., Klanderman, G., Rucklidge, W.: Comparing images using the hausdorff distance. IEEE Transactions on Pattern Analysis and Machine Intelligence 15(9), 850-863 (1993)

18. Goetschalckx, K., Rademakers, F., Bogaert, J.: Right ventricular function by MRI. Curr. Opin. Cardiol., 451-455 (2010) 